1998, Vol. 8, Nos. (1-4), pp. 349-353

Reprints available directly from the publisher

Photocopying permitted by license only
(C) 1998 OPA (Overseas Publishers Association) N.V.

Published by license under the Gordon and Breach Science Publishers imprint. Printed in India.

\title{
A New HEMT Breakdown Model Incorporating Gate and Thermal Effects
}

\author{
LUTFI ALBASHA*, CHRISTOPHER M. SNOWDEN and ROGER D. POLLARD \\ Institute of Microwaves and Photonics, School of Electronic and Electrical Engineering, \\ University of Leeds, Leeds, LS2 9JT, UK
}

\begin{abstract}
This paper presents a comprehensive physical model for the breakdown process in HEMTs. The model is integrated into in a fast quasi-two-dimensional HEMT physical simulator. The work is based on a full study of the complex interactions between the different breakdown mechanisms and the influence of design parameters. The model takes account of tunnelling effects in the region of the gate metallization, and of the thermal effects in the active channel under the gate region.
\end{abstract}

Keywords: Gate tunneling, thermal modelling, breakdown, quasi-two-dimensional modelling

\section{INTRODUCTION}

Microwave circuits such as power amplifiers operate under large-signal conditions. Their ability to perform efficiently is limited by the devices' breakdown characteristics, which limit the transistor's performance and power output. The accuracy of the large-signal design relies on the availability of suitable breakdown models. Popular breakdown theories have not been adequate to independently explain the full picture of the breakdown process in HEMTs. The effects of the gate leakage and substrate conduction in HEMTs on this have not been simulated. Analytical models for ava- lanche breakdown, such as Frensley's [1], were based on physical simulations of the active channel around the gate. The effects of the gate leakage and substrate conduction on this have not been included. This paper considers breakdown more comprehensively including the effects of reverse gate conduction, thermal fluctuations within the active region and substrate conduction [2].

\section{MODEL DESCRIPTION}

The Quasi-Two-Dimensional (Q2D) model used here is based on the earlier work of Snowden [3].

*Corresponding author. Tel.: +44 113 2332082, Fax:+44 113 2332032, email: 1.albasha@elec-eng.leeds.ac.uk. 
The Q2D method is based on the assumption that the fundamental driving force for electron transport is the $x$-directed electric field. The HEMT Q2D model important features have been reported previously [4]. In contrast to MESFETs, spurious substrate current occurs in HEMT buffer layers due to the lateral $E_{x}$ field component. This current is drawn around the depleted channel and reduces the magnitude of the field. It is assumed in this paper that, as a result of the reduction in the electric field, an increase in the breakdown voltage is possible. Figure 1 shows a schematic diagram of a HEMT device.

The computational interpretation of the breakdown model presented in this paper is based on the interactions between the avalanche and gate leakage mechanisms. The relation that both processes simultaneously have with the device design parameters and power dissipation inside the device is included. The flow chart of the model is shown in Figure 2. The gate leakage, conventionally termed 'soft breakdown', is assumed to always occur in devices prior to avalanche. The flow of electrons from the gate into the semiconductor would then influence the impact ionisation process. Adding the leaked electrons to the channel electrons constituting the increasing drain current imposes this effect. The leakage is allowed in the three pinchoff stages.

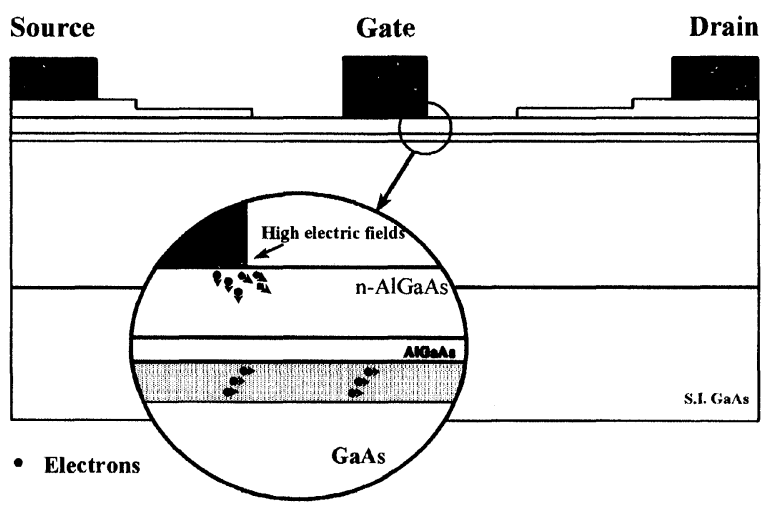

FIGURE 1 Schematic diagram of a HEMT device showing electrons leaking form gate.

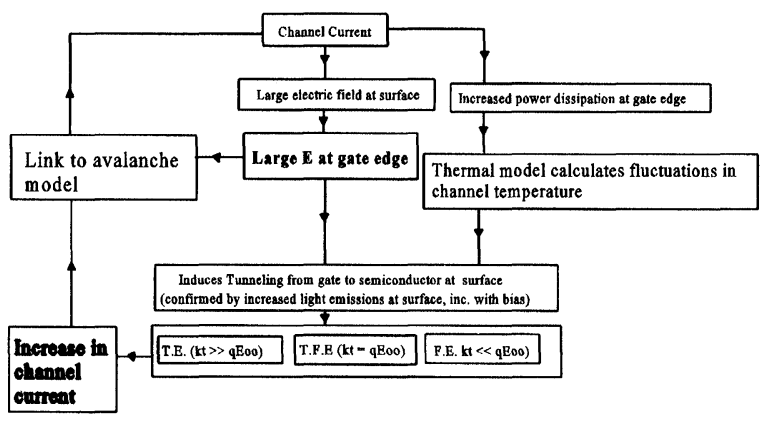

FIGURE 2 Flow chart of the gate breakdown model.

\section{THERMALLY DRIVEN GATE LEAKAGE CURRENT}

The gate leakage model is principally based on a combination of Padovani [5] and Rideout [6] equations. These equations involve complex functions of temperature, barrier height and semiconductor parameters. The form that these equations are presented in the above references is quite complicated. In order to maintain the numerical efficiency of the physical device simulator, new approximations are introduced in this paper, which simplify the tunneling functions and maintain the accuracy of the solution. Tunneling through the gate metal-semiconductor barrier becomes significant in the reverse direction than in the forward direction because the bias voltages involved are usually greater. This cause the potential barrier to become thin enough such that tunneling is dominant. The Thermionic (T), Thermionic-Field (T-F) and Field Emission (FE) gate leakage currents are dependent on the lattice temperature and material specifications. What determines the current mechanism is the temperature of the channel and the applied bias. A new thermal model [7] incorporated into the Q2D physical model computes the instantaneous temperature of the active channel. The output of this model is linked with the breakdown model. This assists the dynamic update of the gate leakage current mechanism. 


\subsection{Thermionic-field Tunneling Current}

This mechanism is the major form of tunneling. The tunneling current is defined by the equation

$$
I_{T-F}=A J_{s} \exp \left(E / \varepsilon^{\prime}\right)
$$

Where $A$ is the gate area, $E$ is applied energy calculated from $q V_{r}, V_{r}$ being the applied reverse bias voltage given a positive sign throughout this work. $q$ is the electron charge and $\varepsilon^{\prime}$ is an energy term defined as:

$$
\varepsilon^{\prime}=E_{o o}\left[\frac{E_{o o}}{K T}-\tanh \left(\frac{E_{o o}}{K T}\right)\right]^{-1}
$$

The term $E_{o o}$ quantifies the diffusion potential from metal into semiconductor. It has two equivalent definitions given in [5] and [6]. The latter definition is adopted in this work, however, slight numeric alteration is needed for consistency of units:

$$
E_{o o}=18.57 \times 10^{-15}\left(\frac{N}{m_{r} \varepsilon_{r}}\right)^{1 / 2}
$$

$N$ is the doping density per $\mathrm{m}^{-3}$. In order to use equation (3) in (2), $E_{o o}$ must be converted back by multiplying by $q$, the electron charge. The type of HEMT used connects the gate metalisation directly to the $N$-doped $\mathrm{AlGaAs}$ layer.

In equation (1), $J_{s}$ is defined as the saturation current given in [5] as:

$$
\begin{aligned}
J_{s} & =\frac{R\left(\pi E_{o o}\right)^{1 / 2}}{K T}\left[q V_{r}+\frac{q \phi_{b}}{\cosh ^{2}\left[\frac{E_{o o}}{K T}\right]}\right]^{1 / 2} \\
& \times \exp \left(\frac{-q \phi_{b}}{E_{o}}\right)
\end{aligned}
$$

where $\phi_{b}$ is the Schottky barrier height. $E_{o}$ is a term defined as:

$$
E_{o}=E_{o o} \operatorname{coth}\left(\frac{E_{o o}}{K T}\right)
$$

It was observed during the course of this work that in the saturation current equation (4), the square root term was dominated by the first term. Hence a simpler approximate numerical expression for the saturation current was deduced:

$$
J_{s}=\frac{R}{k T}\left[\pi E_{o o} q V_{r}\right]^{1 / 2} \exp \left(\frac{-q \phi_{b}}{E_{o}}\right)
$$

which is integrated into the model. An empirical difference limit between equations (4) and (6) was reached after some experimentation's beyond which if this limit is exceeded, the solution obtained from equation (6) was observed to affect the numerical accuracy of the tunneling current. The model then switches to the more stringent expression of equation (4). The thermionic and field emission currents are calculated using similar equations. Threshold equations are incorporated which, according to the thermal status of the device, the appropriate leakage mechanisms is invoked.

\section{SIMULATION RESULTS}

Figure 3 shows the effect of varying the temperature on the tunnelling currents at various

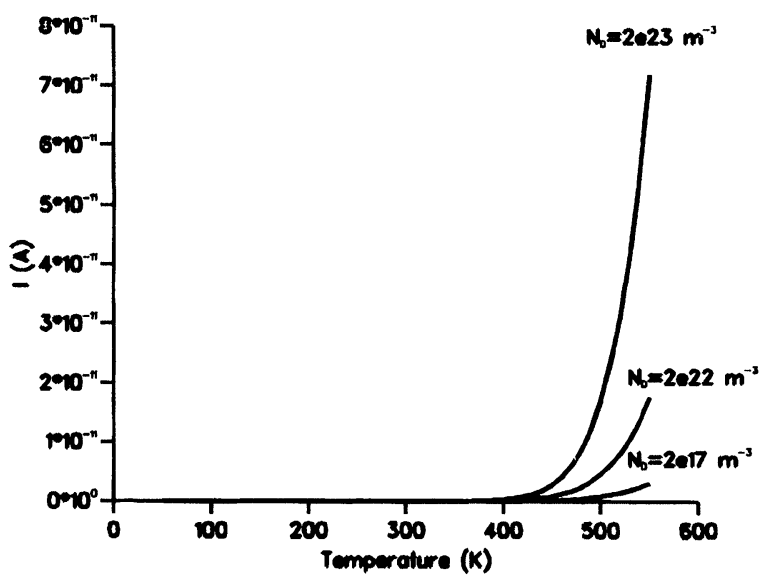

FIGURE 3 Tunneling current versus channel temperature at various doping concentrations. 
doping levels. An increase in the T-F current is observed with an increase in the doping concentration. Figure 4 compares between the thermionic-field and field emission currents with respect to temperature. The T-F current is clearly of more prominent effect. The simulation of the DC I-V characteristics of a HEMT device enabling gate current tunnelling mechanism is shown in Figure 5. The soft breakdown mechanism is influenced by the design parameters and applied

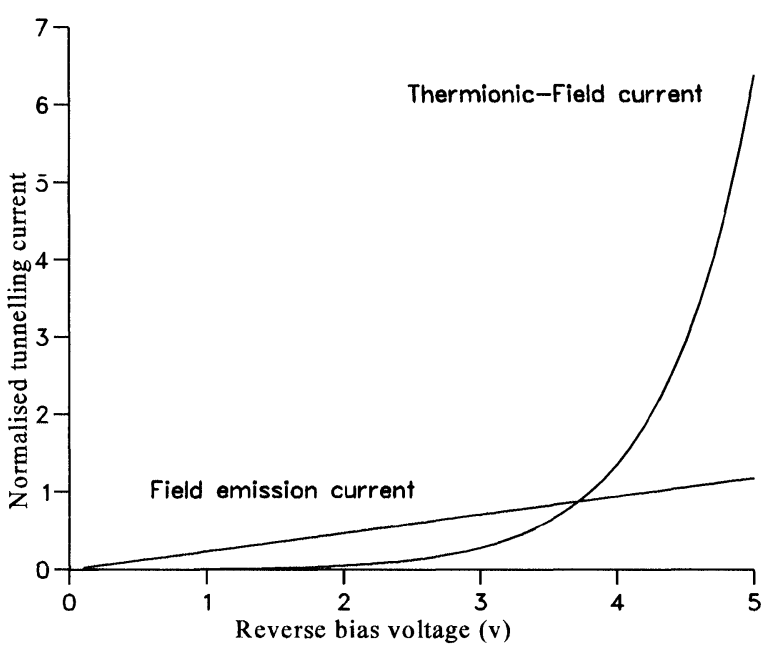

FIGURE 4 Comparison between the thermionic-field and field-emission leakage currents.

\section{Characterstics of a HEMT}

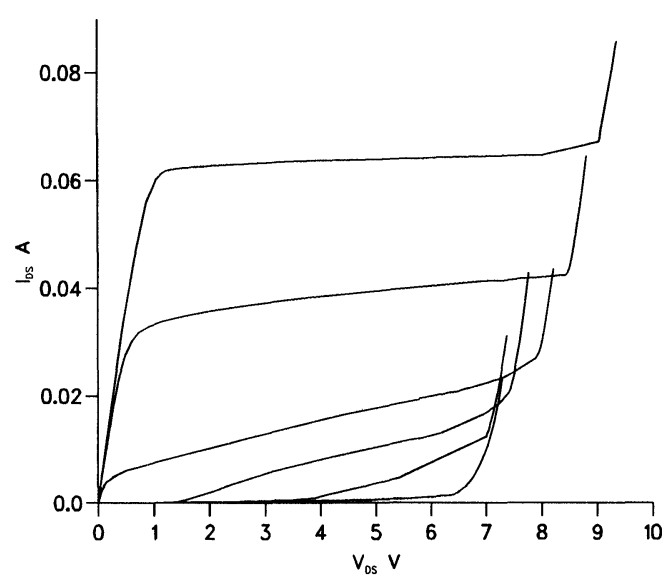

FIGURE 5 I-V characteristics of a HEMT device incorporating the gate model. bias. It can be practically and numerically observed when the stimulating conditions are met. Comparisons with measured data in [8] shows good agreement between simulated and measured results of a MESFET case.

\section{CONCLUSION}

The Q2D physical device simulation of HEMTs has been expanded to include soft thermal breakdown effects using tunnelling current mechanisms. The influence of soft breakdown mechanism on the DC characteristics is influenced by the design parameters and applied bias. The thermal effects in the active channel under the gate region are included. This is conducted using an incorporated thermal model, which calculates the channel temperature and updates the thermal breakdown model.

\section{References}

[1] Frensley, W. R. (1981). "Power-limiting breakdown effects in MESFETs," IEEE Trans., ED-28(8), 962-970.

[2] Morton, C. G., Atherton, J. S., Snowden, C. M., Pollard, R. D. and Howes, M. J. "A large-signal physical HEMT model ," Int. Microwave Symp. MTT-S, 3, 1759-1762, San Francisco.

[3] Snowden, C. M. and Pantoja, R. R. (1989). "Quasi-twodimensional MESFET simulation for CAD", IEEE Trans., ED-36(9), 1564-1574.

[4] Morton, C. G. and Snowden, C. M. "HEMT physical model for MMMIC CAD", Proceedings of 25th European Microwave Conference, Sept. 1995.

[5] Padovani, F. A. and Stratton, R. (1966). "Field and thermionic-field emission in schottky barriers", SolidState Electronics, 9, 695-707.

[6] Crowell, C. R. and Ridout, V. L. (1969). "Normalised thermionic-field (T-F) emission in metal-semiconductor (schottky) barriers", Solid-State Electronics, 12, 89-105.

[7] Johnson, R. G., Snowden, C. M. and Pollard, R. D. (1997). "A Physics Based Electro-Thermal Model For Microwave And Millimetre Wave HEMTs", International microwave symposium, MTT-S, Denver, USA.

[8] Albasha, L., Snowden, C. M. and Pollard, R. D. "Breakdown Characterization of HEMTs and MESFETs Based on A New Thermally Driven Gate Model", International Conference on Simulation of Semiconductors processes and Devices SISPAD, 8-10 Sep. 1997, Cambridge, MA, USA (To be published).

\section{Authors' Biographies}

Lutfi Albasha received his B.Eng. (Hons.) degree in Electronic and Electrical Engineering from the 
University of Leeds in 1990. In 1991 he obtained an M.Sc. (with distinction) in RF communications. In 1992, He joined the Microwave and Terahertz Technology Group at the University of Leeds and obtained his Ph.D. in 1995. His thesis was on the electromagnetic modelling of microwave circuits and its feasibility as a CAD tool. In 1994 he was employed by Filtronics Components Ltd., UK. Currently he is a research fellow at the University of Leeds conducting new studies on the high frequency breakdown modelling in HEMTs and its measurement techniques at W-band frequencies. His research interests include electromagnetic numerical modelling and the design and measurements of microwave circuits and devices.

Christopher M. Snowden received the B.Sc., M.Sc. and Ph.D. degrees from the University of Leeds. After graduating in 1977 he worked as an Applications Engineer for Mullard, Mitcham. His $\mathrm{Ph}$.D. studies were conducted in association with Racal-MESL and were concerned with the largesignal characterisation of MESFET microwave oscillators. In 1982 he was appointed Lecturer in the Department of Electronics at the University of York. He joined the Microwave Solid State Group in the Department of Electrical and Electronic at the University of Leeds in 1983. He now holds the Chair of Microwave Engineering in the Microwave and Terahertz Technology Research Group and is also currently Head of the Department of Electronic and Electrical Engineering. During 1987 he was a Visiting Research Associate at the California Institute of Technology. He has been a Consultant to M/A-COM Inc., Corporate Research and Development since 1989, where he was on sabbatical leave during the period 1990-91. During this year he represented M/A-COM as Senior Staff Scientist. He was Chairman of the 1995 international Microwaves and RF Conference. $\mathrm{He}$ is a Member of the MIT Electromagnetics Academy. He is also a Top Scientist at the International Research Centre for Telecommuni-
cations-Transmission and Radar, Delft University of Technology, Netherlands. Professor Snowden is a Fellow of the IEEE and a Fellow of the IEE. He is a Distinguished Lecturer (1996/7) for the IEEE (Electron Devices Society). He is co-Chairman of the MTT-1 Committee and a Member of the 1997 IEEE MTT-S Technical Program Committee. His main research interests include compound semiconductor device modelling, microwave, terahertz and optical nonlinear subsystem design and advanced semiconductor devices. He has written 7 books and over 190 papers.

Roger D. Pollard was born in London, England in 1946. He received his technical education, graduating with the degrees of $\mathrm{BSc}$ and $\mathrm{PhD}$ in Electrical and Electronic Engineering, at the University of Leeds, Leeds, UK.

He holds the Hewlett-Packard Chair in High Frequency Measurements in the School of Electronic and Electrical Engineering at the University of Leeds where he has been a faculty member since 1974. He is Deputy Director of the Institute of Microwaves and Photonics which has over 40 active researchers, a strong graduate program and has made contributions to microwave passive and active device research. The activity has significant industrial collaboration as well as a presence in continuing eduction through its Microwave Summer School. Professor Pollard's personal research interests are in microwave network measurements, calibration and error correction, microwave and millimetre-wave circuits and large-signal and nonlinear characterization. He has been a consultant to the Hewlett-Packard Company, Santa Rosa, CA since 1981. He has published over 100 technical articles and hold 3 patents.

Roger Pollard is a Chartered Engineer, a member of the Institution of Electrical Engineers (UK) and a Fellow of the IEEE. He is 1998 President of the IEEE Microwave Theory and Techniques Society where he is serving his second term as an elected member Administrative Committee. 

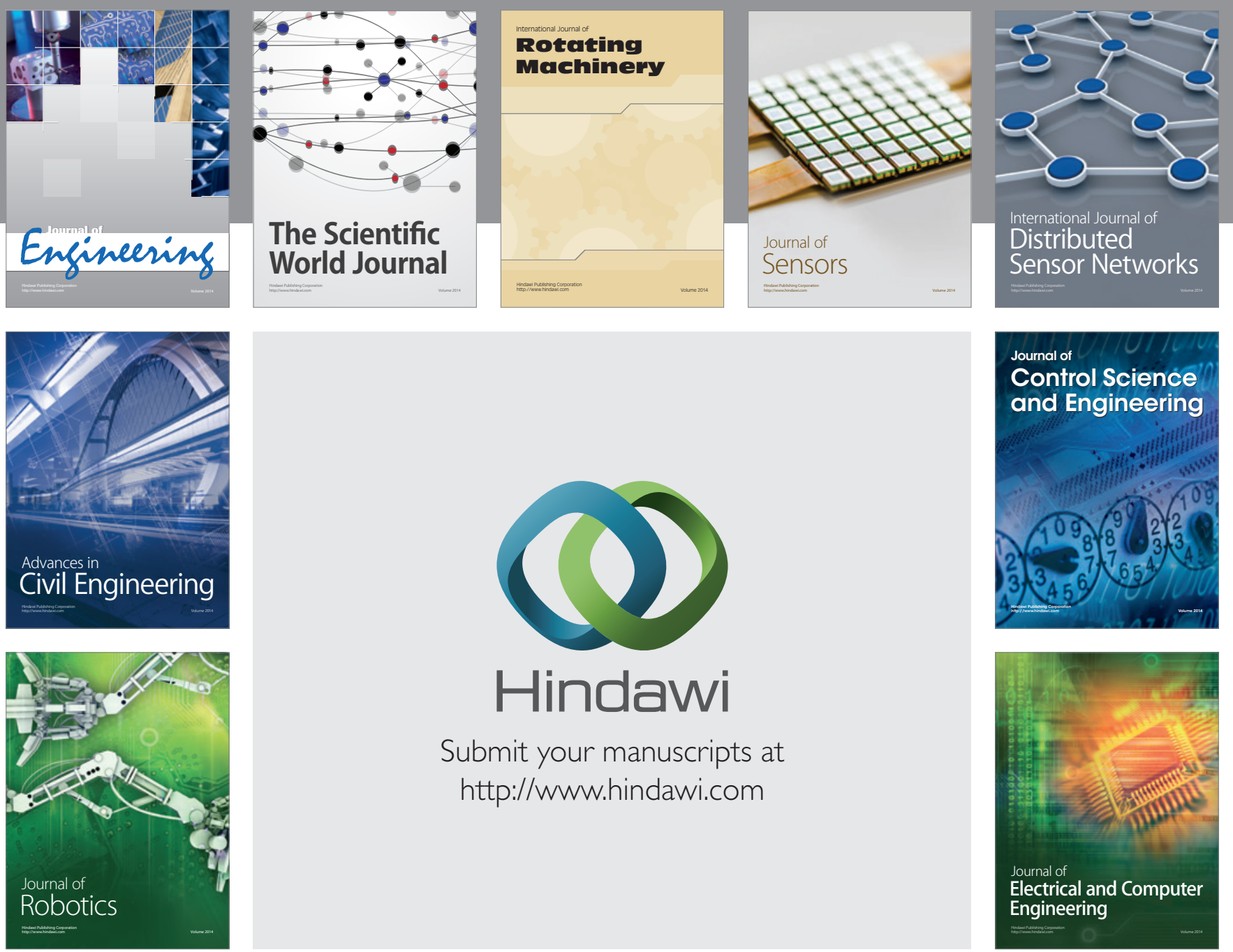

Submit your manuscripts at

http://www.hindawi.com
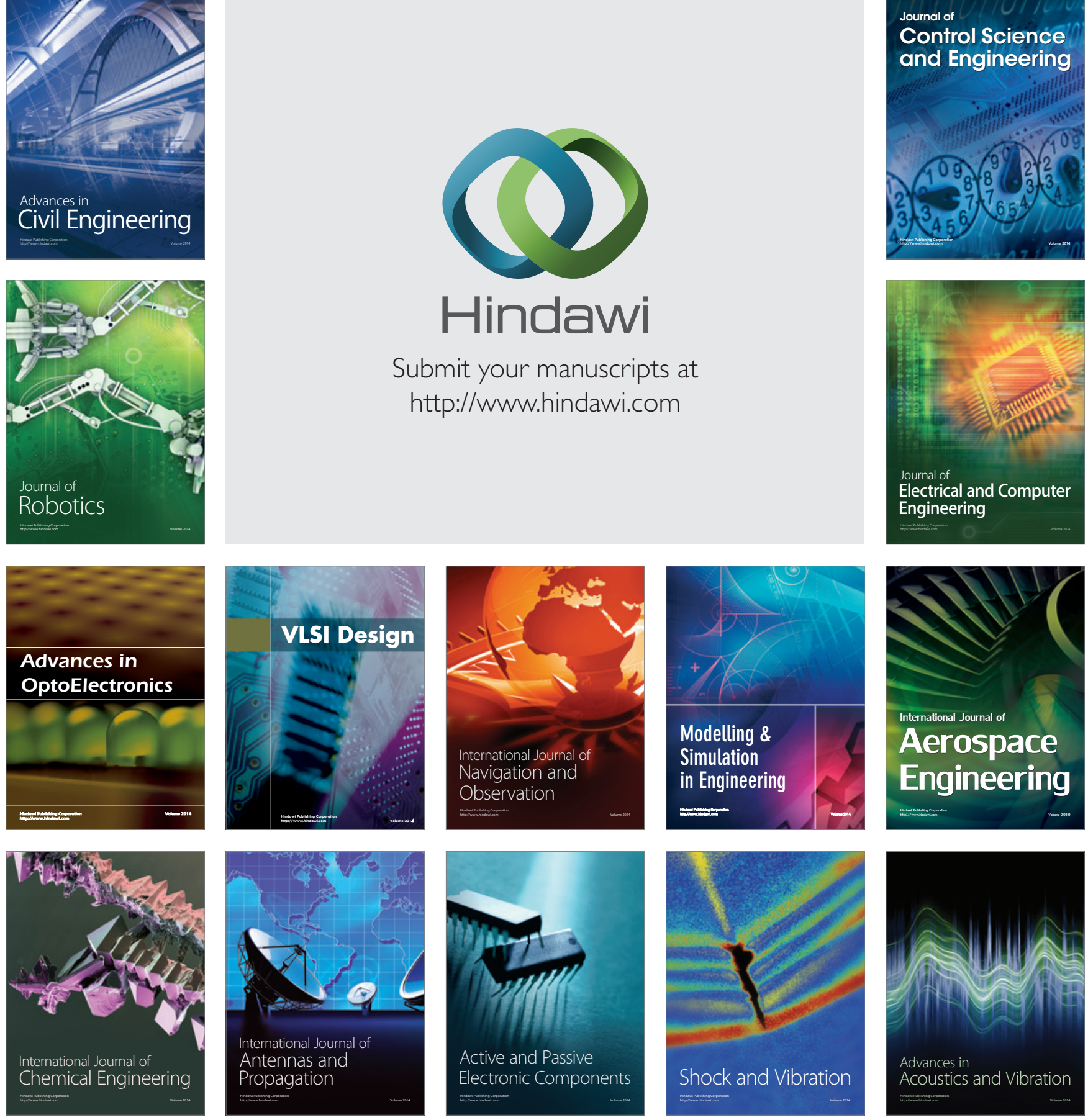